\title{
Prevalence of Diagnostic Errors as Predictors of Obstetric Outcomes among Post-Natal Mothers in Bungoma County, Kenya
}

\author{
David Nandebe Wafula, Bernard Wesonga, and Maximilla Wanzala
}

\section{ABSTRACT}

\begin{abstract}
Diagnosis is the foundation of a correct intervention. However, diagnostic errors result in wrong interventions. This is a global phenomenon, where it is a common problem; which has been understudied. Looking at the Philippines, diagnostic errors are three times most likely to make pregnant women develop obstetric complications. In Kenya, there is a high annual maternal mortality prevalence ratio of $362 / 100,000$ live births, with Bungoma County exceeding the national maternal mortality prevalence ratio of 382/100,000 live births annually. Maternal mortality more often than not, a factor, that arises from morbidity is fuelled by diagnostic errors that required determination of its consequences on obstetric outcomes in Bungoma county. Thus, this study investigated the prevalence of diagnostic errors as predictors of obstetric outcomes among post-natal mothers in Bungoma County. The study employed a descriptive cross-sectional research design, which was hospital-based (Bungoma and Webuye hospitals). Systematic sampling was used to obtain 384 respondents and purposive sampling to select 8 health care workers as key informants. Data was collected using a structured questionnaire and an interview guide. the pre-test was done; validity was established through crosschecking and reliability calculated using the Cronbach method $(0.89)$. Using a statistical package for social sciences version 25 , descriptive statistics were run. The study revealed a prevalence ratio of $\mathbf{3 . 9 9 6}$ whereas delayed diagnosis was $43.1 \%$, missed $38.8 \%$, absent diagnosis $27.5 \%$, wrong diagnosis $34.9 \%$, misinterpretation of results 24.1, unmatched $26.3 \%$ and unnecessary investigation $9.3 \%$. The study demonstrates that a correct diagnosis is a viable strategy in preventing unsafe obstetric outcomes and by extension minimizing morbidity and mortality among pregnant women.
\end{abstract}

Keywords: Diagnosis; Diagnostic Errors; Obstetric outcomes; Maternal Health (morbidity and mortality; Post-Natal.

\section{INTRODUCTION}

According to [1] 600,000 women in the world die each year from pregnancy-related complications and $15 \%$ of all pregnant women develop life-threatening complications (morbidity). A diagnostic error occurs whenever there is a misdiagnosis, missed diagnosis, wrong diagnosis or delayed diagnosis including misinterpretation of radiological and laboratory results [2]. Maternal and perinatal morbidity such as pre-term birth, infection, hypertensive disease, and intrapartum asphyxia are significant causes of unsafe pregnancy outcomes mostly in the global south [3]. Pregnant mothers are at a higher risk of dying during the pregnancy period. Most of the deaths occur around the time of birth, during delivery and immediately after giving birth (postpartum period), with the first 24 hours being the most critical [4]. Ninety-nine per cent of these deaths occurred in developing countries, with Sub Saharan Africa (SSA) region
Submitted : June 17, 2021

Published : July 09, 2021

ISSN: 2593-8339

DOI: 10.24018 /ejmed.2021.3.4.938

David Nandebe Wafula*

Kenya Medical Training College,

Nairobi, Kenya.

(e-mail: nandebedavid ${ }^{\circledR}$ yahoo.com

dnandebe@kmtc.ac.ke)

Benard Wesonga

Masinde Muliro University of Science and Technology, Kakamega, Kenya.

School of Medicine

(e-mail: bwesonga2@gmail.com)

Maximilla Wanzala

Masinde Muliro University of Science

and Technology, Kakamega,

Kakamega County, Kenya.

School of Public Health, Biomedical

Sciences \& Technology

(e-mail: mwanzala@mmust.ac.ke)

*Corresponding author alone accounting for $66 \%$ of these deaths [5]. These deaths do however mask the magnitude of the challenges that women face during the process of pregnancy and childbirth. For any one maternal death, 100 women develop severe maternal morbidity from life-threatening obstetric complications referred to as near misses [6]. Intrapartum care and hypertensive disease remain high priority areas for addressing perinatal mortality in sub-Saharan Africa [3]. The mortalities and morbidities can be significantly minimized if the right diagnosis is made early enough and management or intervention made. If the diagnosis is missed incorrect or a diagnostic error occurred, then an adverse outcome may result [6]. In Kenya matters of maternal mortality rate is of concern too, making it among the top ten countries where maternal mortality is high, and the country still records average maternal mortality of 362 deaths per 100,000 live births [7]. Bungoma County still records a national mortality ratio of 382 maternal deaths per 100,000 live births [8], yet 
collectively the recommended maternal mortality ratio in Kenya should be 147/100,000 live births. Therefore, this led to this study investigating the prevalence of diagnostic errors as predictors of obstetric outcomes in Bungoma County.

\section{Problem Statement}

Diagnostic errors may be significant but less recognized in health care particularly in a birthing facility setting. Looking at the Philippines, the prevalence of diagnostic errors in obstetrics stood at $29.8 \%$ overall and the likelihood odds of those mothers getting complications (morbidity) were 2.96 [9]. In Kenya, diagnostic errors appear to have presented a blind spot in obstetric care and this could as well be contributing to morbidity and mortality. This was evidenced by [6] report of maternal audits that revealed over $75 \%$ of maternal deaths were related to health care worker factors among them diagnostic errors. In Bungoma County, the maternal mortality ratio stands at $382 / 100,000$ whereas the national average is $362 / 100,000$, which is beyond the national target of 147 per 100,000 live births (8). Bungoma County, despite having a maternal mortality ratio of $382 / 100,000$, is not near the national mortality ratio target of 147 per 100,000 live births [10]; which led to this study to investigate the prevalence of diagnostic errors as predictors of obstetric outcomes in Bungoma County.

\section{CONCEPTUAL FRAMEWORK}

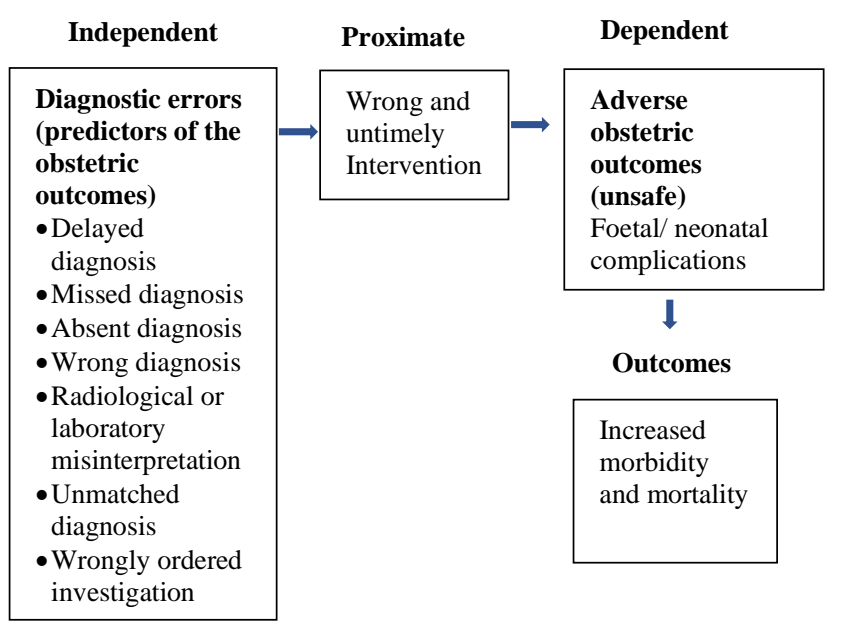

\section{Materials AND Methods}

A cross-sectional research design was used for this study. The design was flexible in describing and analysing the prevalence of diagnostic errors among postnatal mothers at Bungoma county hospitals. The study was carried out at two referral hospitals in Bungoma County which were Bungoma and Webuye. The study targeted all mothers admitted in postnatal wards due to pregnancy and pregnancy complications. What guided the choice of this category of the study population was that these mothers in the postnatal wards had delivered at the facility and had pregnancy or pregnancyrelated complications.

Systematic random sampling was used to get the post-natal mothers in this study where every kth number was picked from the list of mothers on discharge. The post-natal mothers provided their records for verification through content analysis during the interview. Purposive sampling was used to pick the health workers for key informant interview. Quantitative data were collected using structured questionnaires from mothers, while interview guides were used on health workers. Obstetric outcomes information was extracted from the individual mothers, ANC booklet and files through content analysis. Two research assistants were recruited and trained for two days and they assisted in data collection. Sampled mothers were given a questionnaire by the guidance of the research assistants to provide answers and also provided an $\mathrm{MCH}$ booklet for verification in addition to medical records. Healthcare workers were interviewed by lead researcher diagnostic errors leading to obstetric outcome (safe and unsafe).

The researcher coded and entered the data from questionnaires into the computer and analysed using the statistical SPSS version 25. Descriptive statistics were presented in tables in form of proportions. Qualitative data (interviews) were analysed and reported verbatim. Ethical clearance was obtained from Masinde Muliro University of Science and Technology; Institutional Ethics and Review Committee (IERC), Bungoma County Research Committee and National Commission for Science, Technology, and Innovation (NACOSTI). The researcher had to maintain the anonymity and confidentiality of the participants.

\section{RESUlts}

\section{A. Prevalence of Diagnostic Errors at Antenatal Care}

The study sought to investigate the prevalence of diagnostic errors as predictors of obstetric outcomes. To investigate this prevalence, diagnostic error attributes on whether they were exhibited during ANC visits was done. The summary of the responses is shown in Table I. The majority of diagnostic errors were delayed diagnosis 92(28.1\%) with other diagnostic errors being missed diagnosis 56(17.1), absent diagnosis 31(9.5), wrong diagnosis 46 (14.1), misinterpretation of tests 26(8.0), unmatched diagnosis 26(8.0) and unnecessary investigation 27(8.3). The results indicate that during ANC visits, there exist diagnostic errors that may significantly affect obstetric outcomes

TABLE I: DiAgNOSTIC ERRORS AS PREDICTORS OF OBSTETRIC OUTCOMES AT ANTENATAL CARE

\begin{tabular}{cc}
\hline Diagnostic Errors and Obstetric & Percentage (\%) \\
Outcome & $28.1 \%$ \\
Delayed diagnosis & $17.1 \%$ \\
Missed diagnosis & $9.5 \%$ \\
Absent diagnosis & $14.1 \%$ \\
Wrong diagnosis & $8.0 \%$ \\
Misinterpretation of tests & $8.0 \%$ \\
Unmatched diagnosis & $8.3 \%$ \\
Un necessary investigation & \\
\hline $\mathrm{N}=327$. &
\end{tabular}

B. Prevalence of Diagnostic Errors against Obstetric Outcomes

Tables II showed that delayed diagnosis contributed to $43.1 \%$ unsafe obstetric outcomes, missed diagnosis contributed to $38.8 \%$ unsafe obstetric outcomes, absent 
diagnosis contributed to $27.5 \%$ unsafe obstetric outcomes, the wrong diagnosis contributed to $34.9 \%$ unsafe obstetric outcomes, misinterpretation contributed to $24.2 \%$ unsafe obstetric outcomes, unmatched diagnosis contributed to $26.3 \%$ unsafe obstetric outcomes and unnecessary investigation contributed to $19.3 \%$ unsafe obstetric outcomes.

TABLE II: PREVALENCE OF DIAGNOSTIC ERRORS AGAINST OBSTETRIC OUTCOMES

\begin{tabular}{|c|c|}
\hline $\begin{array}{c}\text { Diagnostic Errors and Obstetric } \\
\text { Outcome } \\
\end{array}$ & Percentage \\
\hline \multicolumn{2}{|l|}{ Delayed diagnosis } \\
\hline Unsafe Obstetric outcome & 43.1 \\
\hline \multicolumn{2}{|l|}{ Missed diagnosis } \\
\hline Unsafe Obstetric outcome & 38.8 \\
\hline \multicolumn{2}{|l|}{ Absent diagnosis } \\
\hline Unsafe Obstetric outcome & 27.5 \\
\hline $\begin{array}{l}\text { Safe Obstetric outcome } \\
\text { Wrong diagnosis }\end{array}$ & 72.5 \\
\hline Unsafe Obstetric outcome & 34.9 \\
\hline Safe Obstetric outcome & 65.1 \\
\hline \multicolumn{2}{|l|}{ Misinterpretation of tests } \\
\hline Unsafe Obstetric outcome & 24.2 \\
\hline Safe Obstetric outcome & 75.8 \\
\hline \multicolumn{2}{|l|}{ Unmatched diagnosis } \\
\hline Unsafe Obstetric outcome & 26.3 \\
\hline Safe Obstetric outcome & 73.7 \\
\hline \multicolumn{2}{|l|}{ Unnecessary investigation } \\
\hline Unsafe Obstetric outcome & 19.3 \\
\hline Safe Obstetric outcome & 80.7 \\
\hline $\begin{array}{l}\text { [Safe outcome }=\text { pregnancy had no } \\
\text { pregnancy had complications; the result } \\
\mathrm{N}=327] .\end{array}$ & $\begin{array}{l}\text { unsafe } \\
\text { in pro }\end{array}$ \\
\hline
\end{tabular}

Key informant interviews corroborated these study findings which stated that:

... the solution to reducing diagnostic errors lies in CMEs and well-equipped diagnostic hospitals.

... Use of protocols, good mentorship, feedback on diagnostic errors committed, emphasis on history and proper physical examination may help in minimizing diagnostic errors.

... Some facilities do not have the equipment to monitor pregnancy and some clients cannot afford investigations ordered.

\section{Prevalence Rate of Unmatched and Matched against Safe and Unsafe Obstetric Outcomes}

The researcher investigated the prevalence rate of safe obstetric outcome for both matched and unmatched diagnosis, and the prevalence rate of the unsafe obstetric outcome for both matched and unmatched diagnosis is indicated in Table III. The prevalence ratio of safe obstetric outcome was $=0.975 / 0.244=3.996$. The prevalence ratio of unsafe obstetric outcome was $=0.0253 / 0.7556=0.0335$.

TABLE III: MATCHED AND UNMATCHED DiAgNOSIS AS PREDICTORS OF OBSTETRIC OUTCOMES

\begin{tabular}{cccc}
\hline \multirow{2}{*}{ MCH Booklet } & \multicolumn{3}{c}{ Obstetric Outcome } \\
\cline { 2 - 4 } & Safe & Unsafe & Total \\
\hline Matched diagnosis & $231(70.6 \%)$ & $6(1.8 \%)$ & $237(72.4 \%)$ \\
Unmatched & $22(6.8 \%)$ & $68(20.8 \%)$ & $90(27.6 \%)$ \\
diagnosis & $253(77.4 \%)$ & $74(22.6 \%)$ & $327(100 \%)$ \\
Total &
\end{tabular}

$[\mathrm{MCH}=$ Mother and Child Health Booklet, Matched diagnosis = initial and final diagnosis agreed; Unmatched $=$ Either initial diagnosis or final diagnosis were not in agreement].
Key informant interviews corroborated the findings from this study. The key informant stated that:

... The extent of diagnostic errors seems to be on the rise and is generally associated with the present training of health care providers, lack of basic diagnostic equipment for most health facilities during focused antenatal care such as ultrasound.

... Any single diagnostic errors expose a pregnant woman and her baby to a high risk of complication and possible death.

... Correct diagnosis improves the pregnancy outcome, lowers the cost and prevents the cost. Initial diagnosis and final diagnosis are up to almost $10 \%$ due to poor history taking and irrelevant investigations.

... With diagnostic error, a client is given a wrong intervention leading to an effect on the pregnancy. A common misdiagnosis occurs in pre-eclampsia where it is confused for hypertension.

\section{DISCUSSION}

This study employed the use of the Postulated Theoretical Framework (11) that envisaged to understand the variables that contributed to increased diagnostic errors which were predictors of obstetric outcomes in health facilities in Bungoma County. According to [10], the maternal mortality ratio was 382/100,000 live births annually. This number was above Kenya's average annual maternal mortality ratio of $362 / 100,000$. The researcher investigated the variables that were contributing towards increased diagnostic errors in Bungoma, which were: delayed diagnosis, missed diagnosis, absent diagnosis, the wrong diagnosis, misinterpretation, unmatched diagnosis and unnecessary investigation.

It was established that delayed diagnosis contributed $43.1 \%$ to unsafe obstetric outcomes and $56.9 \%$ to a safe obstetric outcome. The delayed diagnosis was established as the main predictor of unsafe obstetric outcomes among pregnant women in Bungoma County. Prompt diagnosis of a condition is at the epicentre of reproductive health. Failure to promptly diagnose a condition that might develop into an adverse condition predisposes the pregnant women towards a negative and riskier obstetric outcome that might also be fatal. This study found out that most pregnant women who developed adverse conditions during pregnancy and even fatalities among them were contributed by delayed diagnosis.

Missed diagnosis contributed $38.8 \%$ to an unsafe obstetric outcome and $61.2 \%$ to a safe obstetric outcome. Professionalism in dealing with pregnancy and pregnant women is paramount to achieving Sustainable Development Goal 3 (SDG\#3). Missed diagnosis during ANC and natal potentially may lead to adverse outcomes that go against SDG\#3. The researcher posited that missed diagnosis contributed to an increased maternal mortality ratio of $382 / 100,000$ in Bungoma County. Besides, absent diagnosis contributed $27.5 \%$ to unsafe obstetric outcomes, with about $72.5 \%$ safe obstetric outcome. Hidden conditions give a false negative medical diagnosis which contributes towards wrong medical conclusions. At Bungoma County, it was established that it contributed to about a quarter of the unsafe obstetric outcomes in Bungoma County. This also meant that there was no retesting of these false-negative to try to manage the 
prevailing conditions that might be accessed from the pregnant woman's medical records and home history.

It was also established that the wrong diagnosis contributed $34.9 \%$ to unsafe obstetric outcomes and $65.1 \%$ to the safe obstetric outcome. The wrong diagnosis has adverse obstetric outcomes in most cases. With the wrong diagnosis, wrong intervention might be applied and fatalities therein. Basing on the maternal mortality ratio of $382 / 100,000$ live births in Bungoma, the wrong diagnosis will continue to increase affecting Kenya's target on the national maternal mortality ratio of 147/100,000 [10]. The increased prevalence of maternal mortality ratio in Bungoma County could be the product of the wrong diagnosis which was noted that the wrong diagnosis was the silent killer and might have claimed the lives of many pregnant women after wrong treatment [12]. The findings on the wrong diagnosis were in support of [13] where it was stated that 12 million people in the USA were misdiagnosed annually. Misinterpretation of diagnostic data contributed $24.2 \%$ to unsafe obstetric outcomes and $75.8 \%$ to the safe obstetric outcome. Lack of specialised treatment at the ANC and maternity is catastrophic to pregnant women. Also, inadequate experience in reproductive health providers working with pregnant women contributes to misinterpretation of the diagnostic data at ANC and maternity. According to [14], diagnostic errors in many instances were caused by failures in the interpretation of the findings mostly radiological and laboratory information. In 2016, the World Health Organisation reported the importance of addressing morbidity and mortality among pregnant women and their foetus/babies (15).

The unmatched diagnosis among the pregnant women contributed $26.3 \%$ to unsafe obstetric outcomes and $63.7 \%$ to the safe obstetric outcome. This was attributed to either wrong initial or wrong final diagnosis or both were wrongly done after the researcher reviewed the medical records of the participants (MCH Booklet and medical notes). The unmatched cases emanated from the unprofessionalism of the healthcare workers at the ANC and maternity. This was an unfortunate occurrence considering the global campaigns on the promotion of safe motherhood outcome. It was established that unnecessary investigation of the pregnancy progress contributed $19.3 \%$ to unsafe obstetric outcomes and $80.7 \%$ to the safe obstetric outcome. This led to interference with the pregnancy and creating new potential adverse conditions among pregnant women.

In general, the prevalence of safe obstetric outcomes amongst pregnant women without diagnostic errors was 0.975 whereas safe obstetric outcomes among pregnant women with diagnostic errors were 0.244 , which gave a prevalence ratio of 3.995. On the other hand, the prevalence of unsafe obstetric outcomes amongst pregnant women without diagnostic errors was 0.0253 whereas safe obstetric outcomes among pregnant women with diagnostic errors were 0.7556 , which gave a prevalence ratio of 0.0335 . While most pregnancies and births are uneventful, all pregnancies are at risk. Failure to properly diagnose a condition contributed to about $58 \%$ of errors in emergency departments that rippled to obstetric emergencies. Diagnostic errors receive comparatively less attention and yet they were common [5]. The World Health Organisation found that around $15 \%$ of all pregnant women develop a potentially life- threatening complication that calls for skilled care, some requiring a major obstetrician intervention to survive [4]. Pregnancy is double-edged - joyful anticipatory time and on the flip side grave concern and anxious time.

\section{CONCLUSION}

The delayed diagnosis, missed diagnosis and wrong diagnosis were the main diagnostic errors that contributed to increased unsafe obstetric outcomes in Bungoma County. The unmatched diagnosis during antenatal care visits resulted in a higher prevalence of diagnostic errors among postnatal mothers at Bungoma County hospitals in Kenya. It was established that the prevalence ratios for safe obstetric outcome were 3.996 while for unsafe outcome was 0.0335 in Bungoma County hospitals.

\section{RECOMMENDATION}

The ministry of health (MOH) together with county governments consider the possibility of expanding diagnostic capacity including laboratory services and point of use tests in dispensaries, health centres, sub-county hospitals and county hospitals and intensify human resource upgrading through continuing medical education. Health care providers should undergo mandatory updates especially in emergency obstetric diagnosis and neonatal care.

\section{ACKNOWLEDGEMENT}

I acknowledge the almighty God who has been my pillar throughout this study. I owe gratitude to all the women and health workers who were my respondents during the study. I applaud the research assistants in Bungoma and Webuye Hospitals; Inviolata Makhanu, Fatuma, Pamela Pwaipwai and Leonard Lusenaka who tirelessly worked hard during COVID 19 pandemic but ensured the study ran smoothly. I thank my supervisors Dr Benard Wesonga and Dr Maximilla Wanzala for their relentless support and commitment towards making sure I finish the work on time through their diligence supervision. I wish to salute Mr Paul Kem and all the staff of the following hospitals for their support, Bungoma hospital, Webuye Hospital and Kimilili Sub County Hospital. Finally, I also wish to appreciate my family and classmates for their encouragement and support.

\section{REFERENCES}

[1] Ministry of Health (2017), [Kenya]. Saving Mothers lives. First confidential report into maternal deaths in Kenya.

[2] McGlynn EA, McDonald KM, Cassel CK. Measurement is essential for improving diagnosis and reducing diagnostic error: a report from the Institute of Medicine. Jama. 2015 Dec 15;314(23):2501-2.

[3] Allanson ER, Muller M, Pattinson RC. Causes of perinatal mortality and associated maternal complications in a South African province: challenges in predicting poor outcomes. BMC pregnancy and childbirth. 2015 Dec;15(1):1-7.

[4] World Health Organization. Medication safety in polypharmacy technical report. World Health Organization; 2019.

[5] Nathan, R. O., Swanson, J. O., Swanson, D. L., McClure, E. M., Bolamba, V. L., Lokangaka, A., \& Goldenberg, R. L. (2017) Evaluation of focused obstetric ultrasound examinations by health care personnel in the Democratic Republic of Congo, Guatemala, Kenya, 
Pakistan, and Zambia. Current problems in diagnostic radiology, 46(3), 210-215.

[6] Ministry of Health [Kenya]. (2017). Bungoma county referral strategy and investment plan 2016/17-2020/21. Nairobi: MoH.

[7] National Council for Population and Development (NCPD) and UNFPA (2013) Kenya Country Office, Kenya Population Situation Analysis (Nairobi: NCPD. and UNFPA, 2013).

[8] Gacheri A. Tackling high maternal deaths in Kenya. Policy Brief, Parliament of Kenya.

[9] Shimkhada, R., Solon, O., Tamondong-Lachica, D., \& Peabody, J. W. (2016). Misdiagnosis of obstetrical cases and the clinical and cost consequences to patients: a cross-sectional study of urban providers in the Philippines. Global health action, 9(1), 32672.

[10] Kenya Demographic and Health Survey (KDHS 2014) Kenya, Ministry of Health.

[11] Singh H. Editorial: (2014). Helping health care organizations to define diagnostic errors as missed opportunities in diagnosis. Joint Commission Journal on Quality\& Patient Safety. ;40(3):99-101. [PubMed]Stud HSO\&P 2001; 17:97-129

[12] Oketch, A (2017). Doctors cite the wrong diagnosis as a silent killer in Kenya, National News. Daily Nation of Thursday 29th 2017

[13] Liberman, A. L., \& Newman-Toker, D. E. (2018). Symptom-Disease Pair Analysis of Diagnostic Error (SPADE): a conceptual framework and methodological approach for unearthing misdiagnosis-related harms using big data. BMJ quality \& safety, 27(7), 557-566.

[14] Graham WJ, Bell JS, Bullough CH. Can skilled attendance at delivery reduce maternal mortality in developing countries?. Safe motherhood strategies: a review of the evidence. 2001.

[15] World Health Organization. Errors Medication. Technical series on safer primary care. Geneva: World Health Organization. 2016.

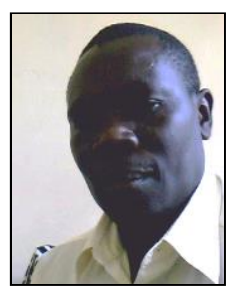

Wafula Nandebe David is a renowned Clinician and Medical Teacher at Kenya Medical Training College, Nairobi Department of Clinical Medicine. 